\title{
Life Sciences Identifier
}

National Cancer Institute

\section{Source}

National Cancer Institute. Life Sciences Identifier. NCI Thesaurus. Code C47841.

A unique identifier meant to be a common way to identify and access biologically significant data, whether that data is stored in files, relational databases, in applications, or in internal or public data sources. Every LSID consists of up to five parts: the Network Identifier (NID); the root DNS name of the issuing authority; the namespace chosen by the issuing authority; the object id unique in that namespace; and an optional revision id for storing versioning information. Each part is separated by a colon. 